Printing low-voltage dielectric elastomer actuators

Alexandre Poulin, Samuel Rosset, and Herbert R. Shea

Applied Physics Letters 107 , 244104 (2015)

Copyright (2015) AIP Publishing. This article may be downloaded for personal use only. Any other use requires prior permission of the author and AIP Publishing.

The following article appeared in : Applied Physics Letters 107 , 244104 (2015) and may be found at http://dx.doi.org/10.1063/1.4937735 


\title{
Printing low-voltage dielectric elastomer actuators
}

\author{
Alexandre Poulin, ${ }^{\text {a) }}$ Samuel Rosset, and Herbert R. Shea ${ }^{\text {b) }}$ \\ Microsystems for Space Technologies Laboratory, Ecole Polytechnique Fédérale de Lausanne (EPFL), \\ 2002 Neuchâtel, Switzerland
}

(Received 15 September 2015; accepted 1 December 2015; published online 16 December 2015)

\begin{abstract}
We demonstrate the fabrication of fully printed thin dielectric elastomer actuators (DEAs), reducing the operation voltage below $300 \mathrm{~V}$ while keeping good actuation strain. DEAs are soft actuators capable of strains greater than $100 \%$ and response times below $1 \mathrm{~ms}$, but they require driving voltage in the $\mathrm{kV}$ range, limiting the possible applications. One way to reduce the driving voltage of DEAs is to decrease the dielectric membrane thickness, which is typically in the $20-100 \mu \mathrm{m}$ range, as reliable fabrication becomes challenging below this thickness. We report here the use of pad-printing to produce $\mu \mathrm{m}$ thick silicone membranes, on which we pad-print $\mu \mathrm{m}$ thick compliant electrodes to create DEAs. We achieve a lateral actuation strain of $7.5 \%$ at only $245 \mathrm{~V}$ on a $3 \mu \mathrm{m}$ thick pad-printed membrane. This corresponds to a ratio of $125 \% / \mathrm{kV}^{2}$, by far the highest reported value for DEAs. To quantify the increasing stiffening impact of the electrodes on DEA performance as the membrane thickness decreases, we compare two circular actuators, one with $3 \mu \mathrm{m}$ - and one with $30 \mu \mathrm{m}$-thick membranes. Our experimental measurements show that the strain uniformity of the $3 \mu \mathrm{m}$-DEA is indeed affected by the mechanical impact of the electrodes. We developed a simple DEA model that includes realistic electrodes of finite stiffness, rather than assuming zero stiffness electrodes as is commonly done. The simulation results confirm that the stiffening impact of the electrodes is an important parameter that should not be neglected in the design of thin-DEAs. This work presents a practical approach towards low-voltage DEAs, a critical step for the development of real world applications. @ 2015 AIP Publishing LLC. [http://dx.doi.org/10.1063/1.4937735]
\end{abstract}

Dielectric elastomer actuators (DEAs) are soft stretchable transducers that consist of an elastomer membrane sandwiched between two compliant electrodes. When a voltage difference is applied between the electrodes, an electrostatic force is generated, squeezing the membrane. Since elastomers are incompressible, this out-of-plane compression is accompanied by an in-plane expansion (Fig. 1(b)). Actuation strains greater than $100 \%$ (Ref. 1) and sub-ms response time $^{2}$ can be achieved with DEAs, which find applications in a wide range of fields including soft-robotics, ${ }^{3-5}$ optics, ${ }^{2,6,7}$ and microfluidics. ${ }^{8-10}$ DEAs are well suited to miniaturisation, as power density is high, ${ }^{1}$ and complex shapes and systems can be made using simple architectures. DEAs however require voltages in the $\mathrm{kV}$ range, which limits potential applications, due to possible safety concerns but mostly due to the cost and size of the $\mathrm{kV}$ control electronics.

Significant efforts have been made to decrease the driving voltage of DEAs while maintaining good actuation performance. For small deformations and ignoring the stiffening impact of the electrodes, the lateral $s_{x}=s_{y}$ and vertical $s_{z}$ strains can be approximated by ${ }^{11}$

$$
s_{x}=-\frac{s_{z}}{2}=\epsilon \frac{E^{2}}{2 Y}=\epsilon \frac{V^{2}}{2 t_{m}^{2} Y},
$$

where $\epsilon$ is the dielectric permittivity of the membrane, $Y$ is its Young's modulus, $E$ is the electric field between the two electrodes, $V$ is the applied voltage, and $t_{m}$ is the membrane

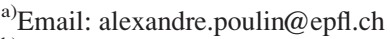

b)Laboratory website: http://lmts.epfl.ch
}

thickness. Thus, from Eq. (1), the operation voltage can be reduced by optimizing the material properties $\epsilon$ and $Y$, or reducing $t_{m}$.

Lower Young's modulus can be achieved by decreasing the cross-link density ${ }^{12}$ or by adding plasticizers ${ }^{13}$ to the elastomer. This approach however decreases the mechanical robustness and the energy density of the materials. The dielectric permittivity can be adjusted by adding different fillers ${ }^{14,15}$ or by functionalizing the elastomer. ${ }^{14,16}$ It is only recently that a high permittivity elastomer with good mechanical properties was realised, ${ }^{16}$ enabling the fabrication of an actuator working at only $10 \mathrm{~V} / \mu \mathrm{m}$, while DEAs traditionally require electric fields over $100 \mathrm{~V} / \mu \mathrm{m}$. Nevertheless, the actuator was based on $180 \mu \mathrm{m}$-thick membranes and still required $1 \mathrm{kV}$ to reach $7 \%$ of lateral strain.

Membrane thickness can be reduced by prestretching, or the membrane can be manufactured directly with the desired dimensions. The later being a more suitable approach for DEAs since it affects only $t_{m}$, unlike pre-stretching which also modifies $Y .{ }^{17}$ Typical DEAs have membranes in the 20 to $100 \mu \mathrm{m}$-range. Below those thicknesses, the fabrication becomes challenging and membrane quality is critical given the very large electric fields applied. Techniques such as spin-coating and blade-casting can be used to make sub $\mu \mathrm{m}$ membranes, ${ }^{18}$ but not in a process, nor with breakdown strength compatible for use in DEAs. The use of molecular beam deposition was reported for the fabrication of a bending DEA with a $200 \mathrm{~nm}$ thick silicone membrane. ${ }^{19}$ The actuation strains achieved with this actuator are however very limited, mainly because of the large stiffening impact of the sputtered Au electrodes. ${ }^{20}$ 


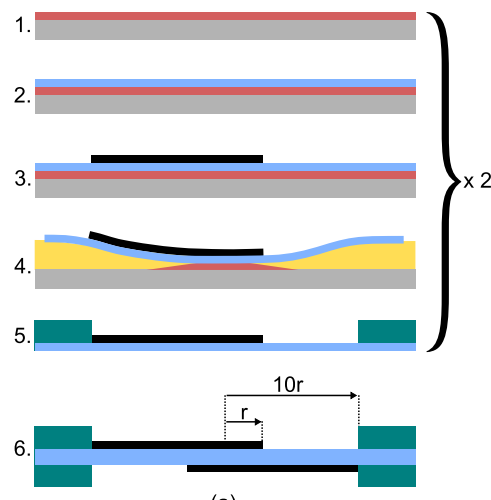

(a)

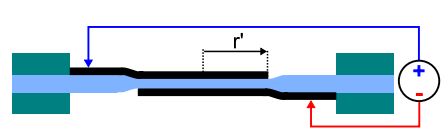

(b)

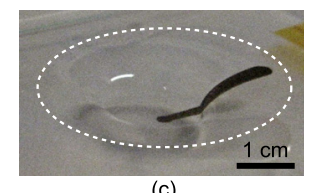

(c)

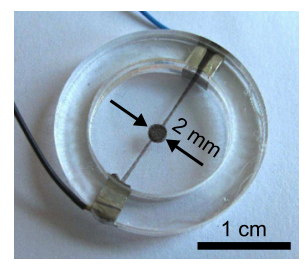

(d)

: Polyethylene terephthalate (PET) foil 드: Poly(acrylic) acid (PAA) layer

: Stretchable PMDS-based electrodes Polydimethylsiloxane (PDMS) : Demineralized water

: Poly(methyl methacrylate) (PMMA)

FIG. 1. (a) Fabrication process for printing thin DEAs with membrane thicknesses in the $\mu$ m-range. (b) The active area defined by the overlapping electrodes expands in-plane when a voltage difference is applied. (c) Picture of the membrane during release (step 4 of the process flow); the dotted line indicates the boundary of the floating transparent membrane. (d) Picture of the completed DEA with a $3 \mu \mathrm{m}$-thick membrane.

In this work, we present a fabrication method to print thin DEAs, reducing the operation voltage below $300 \mathrm{~V}$ while keeping good actuation strains. Using pad-printing, we produced $\mu \mathrm{m}$ thick silicone elastomer membranes, on top of which we pad-printed stretchable electrodes. The thickness of the electrodes is in the $\mu \mathrm{m}$ range and was kept constant, its value being determined by the printing technique and by the desired electrical conductivity. We report a lateral actuation strain of $7.5 \%$ at only $245 \mathrm{~V}$, achieved on a DEA made of a $3 \mu \mathrm{m}$ thick membrane. To observe the increasingly important mechanical effect of electrodes as the membrane thickness decreases, we compare two circular actuators, one with $3 \mu \mathrm{m}$ and one with $30 \mu \mathrm{m}$-thick membranes. The results show that the electrodes stiffening impact is an important design parameter that should not be neglected for thin DEAs.

The fabrication of ultra-thin DEAs presents important technical challenges, namely, (a) producing thin and uniform elastomer membranes, and (b) patterning stretchable electrodes on the very soft membranes without damaging them or adding stiffness. We report here a unique fabrication process that overcomes those difficulties using pad-printing, a commercially available technology. Figure 1(a) details the fabrication steps: 1. A solution of 5\% poly(acrylic) acid (PAA), 15\% water, and $80 \%$ isopropanol is applied by blade casting (ZAA 2300, Zehntner GmbH, Switzerland) on a high quality film of Polyethylene terephthalate (PET). The solvents quickly evaporate at room temperature, leaving a smooth PAA sacrificial layer on top of the PET film. 2. A thin film of silicone elastomer (Sylgard 184, Dow Corning, USA) is stamped (TMP 101, Teca-Print AG, Switzerland) in the shape of a $3 \mathrm{~cm}$ wide circle on top of the PAA layer and cured at $80^{\circ} \mathrm{C}$ for $1 \mathrm{~h}$. 3. A stretchable electrode made of a carbon black-elastomer composite is stamped on top of the silicone film and cured for $30 \mathrm{~min}$ at $80^{\circ} \mathrm{C} .^{21} 4$. The membrane is released from the PET substrate by dissolving the sacrificial layer in warm demineralized water. As seen in Fig. 1(c), the membrane floats at the surface of the water bath as it gradually releases from the substrate. 5. After release, the membrane is dried, stretched, and fixed to a rigid Poly(methyl methacrylate) (PMMA) frame. The resulting suspended membrane constitutes one half of the actuator. 6. Two membranes are assembled to complete the DEA. A drop of ethanol is used between the membranes during the assembly in order to help with the alignment and to ensure a uniform contact.

A picture of the final device is presented in Fig. 1(d), where the stretchable electrodes appear in black. They have a circular geometry that expands in-plane when actuated (Fig. 1(b)), a standard design for DEA performance testing. ${ }^{1}$ The initial radius $\mathrm{r}=2 \mathrm{~mm}$ of the active area is ten times smaller than the inner radius of the PMMA frame, and the device is under an equibiaxial prestretch of $\lambda=1.1$. The membrane is only $3 \mu \mathrm{m}$-thick after prestretch, thus slightly thicker than the electrodes that were measured to be between $1 \mu \mathrm{m}$ - and $2 \mu \mathrm{m}$-thick. For comparison, a second actuator was fabricated using our standard DEA fabrication process. ${ }^{21}$ This reference DEA is based on exactly the same materials and geometry, except for having a $30 \mu \mathrm{m}$-thick membrane. We refer to the two devices as the $3 \mu \mathrm{m}$-DEA and $30 \mu \mathrm{m}$ DEA.

Figure 2 presents the measured Strain-Voltage curves for the $3 \mu \mathrm{m}$-DEA and the $30 \mu \mathrm{m}$-DEA. The average lateral strain corresponds to a percentage change of the radius of the circular active area. The driving voltage to reach $7.5 \%$ strain is more than 10 times lower for the thinner DEA, reduced from $3.3 \mathrm{kV}$ to only $245 \mathrm{~V}$. An intuitive performance metric for low-voltage DEAs would be the strain-to-voltage ratio $\left(s_{x} / \mathrm{V}\right)$, measuring how much strain is generated per volt. Eq. (1) however shows that the $s_{x} / V$ ratio varies with the driving voltage, which can easily lead to ambiguous comparisons between actuators if they are not tested at the same voltage. We find that the strain-to-voltage-squared ratio $\left(s_{x} / V^{2}\right)$ is a more useful performance metric in the frame of this work. Eq. (1) shows that the $s_{x} / V^{2}$ ratio is a device-constant parameter, varying only with the material properties and the device geometry. Using this metric as a figure of merit, the actuator performance between the 3 and $30 \mu \mathrm{m}$ devices improves by more than two orders of magnitude, from $0.7 \% / \mathrm{kV}^{2}$ for $30 \mu \mathrm{m}$ to $124.9 \% / \mathrm{kV}^{2}$ at $3 \mu \mathrm{m}$. This is more than one order of magnitude higher than any other value reported for DEAs. State-ofthe-art devices achieving area strain as high as $488 \%,{ }^{22}$ and operating at electric field as low as $10 \mathrm{~V} / \mu \mathrm{m},{ }^{16}$ exhibit strain-

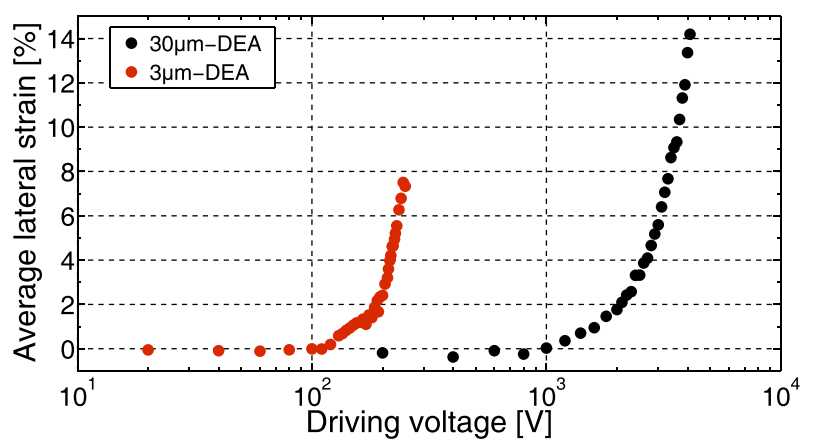

FIG. 2. The actuation voltage for the $3 \mu \mathrm{m}$-DEA is more than 10 times lower than for the $30 \mu \mathrm{m}$-DEA. The average lateral strain corresponds change in radius of the active area. 
to-voltage-squared ratio of only $5.7 \% / \mathrm{kV}^{2}$ and $7 \% / \mathrm{kV}^{2}$, respectively.

It can be seen from Fig. 2 that the maximum actuation strain decreases by almost a factor 2 for the thinner DEA. The operation range was limited by dielectric breakdown at $7.5 \%$ strain for the $3 \mu \mathrm{m}-\mathrm{DEA}$, and by loss of mechanical tension at $14.2 \%$ strain for the $30 \mu \mathrm{m}$-DEA. The reduced actuation strain obtained with the thinner DEA is however largely compensated by a 10 times lower driving voltage. In addition, a lateral strain of $7.5 \%$ is enough to meet the requirements of many DEA applications. ${ }^{2,9}$ Two main effects can explain reduced actuation strain for the $3 \mu \mathrm{m}$ DEA. The first one is non-uniformities in the membrane leading to premature local dielectric breakdown. The nonuniformities can be thickness variations across the membrane or the presence of imperfections such as air bubbles or foreign particles. As characteristic dimensions of the imperfections do not scale down with the membrane thickness, their relative impact increases for thin DEAs. The second effect is the mechanical impact of the electrodes. As the DEA membrane gets thinner, the stiffening impact of the electrodes becomes more important, assuming that the electrodes thickness does not scale down with the membrane.

Strain measurements such as the ones presented in Fig. 2 are usually made by tracking the electrodes boundary or by measuring change in electrode area. Those techniques are quick and easy to implement but only provide an average strain value which can hide valuable information. In order to gain a better insight into the actuation performance of padprinted DEAs, we measured the 2D strain distribution across the electrodes. Pictures of the devices at rest and under actuation were recorded and post-processed using a Matlabbased algorithm that we developed. The algorithm discretizes the images into an array of $n_{x}$ by $n_{y}$ elements. The $\mathrm{x}$ - and $\mathrm{y}$-displacement of each element in the array is then measured by using normalized cross-correlation to match the two images. The area strain and the corresponding lateral strain are calculated for each element by using the displacement values of the closest neighbors. The measurement is performed without adding any markers. The electrode microstructure contains enough visual information for optical cross-correlation to be performed.

Measurements of strain-distribution on the $3 \mu \mathrm{m}$-DEA and the $30 \mu \mathrm{m}$-DEA are presented in Fig. 3. The devices were, respectively, actuated at $245 \mathrm{~V}$ and $3.3 \mathrm{kV}$ which corresponds to an average lateral strain of $7.5 \%$ (Fig. 2). While the $30 \mu \mathrm{m}$-DEA exhibits excellent strain uniformity, the $3 \mu \mathrm{m}$-DEA shows variations over the device area. Due to the membrane incompressibility, high-strain regions are exposed to larger electric fields and can induce premature dielectric breakdown. It is therefore important for the strain-distribution to be as uniform as possible in order to maximize actuator performance. The non-uniform strain profile of the $3 \mu \mathrm{m}$-DEA can have two main origins. The first is the presence of thickness non-uniformities over the membrane, which results in local strain variations. The second is the mechanical impact of the electrodes which have a stiffness similar to the $3 \mu \mathrm{m}$ thick membrane. As a result, the electrodes geometry and uniformity can significantly
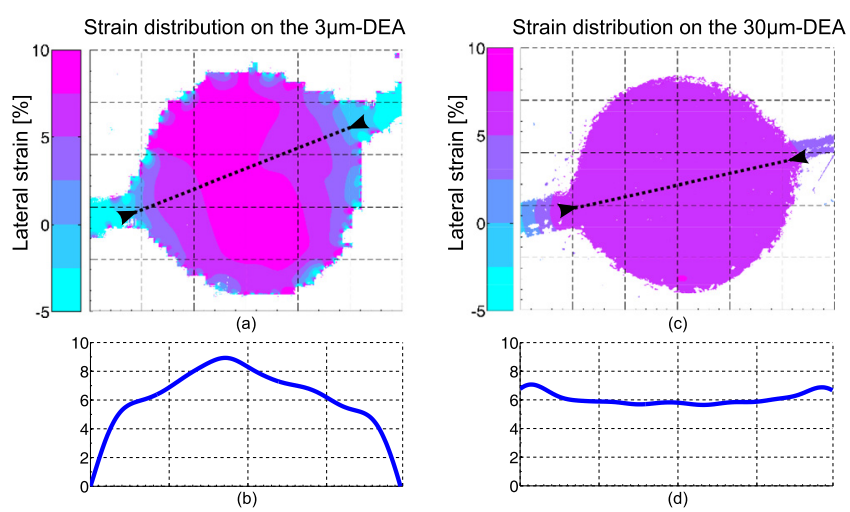

FIG. 3. (a) Lateral strain distribution and (b) cross-section along the black dotted line for the $3 \mu \mathrm{m}$-DEA. (c) Lateral strain distribution and (d) crosssection along the black dotted line for the $30 \mu \mathrm{m}$-DEA. The stiffening impact of the electrodes induces high-strain regions on the $3 \mu \mathrm{m}$-DEA. Those regions experience higher electric field and can therefore induce premature dielectric breakdown.

affect the strain profile. Although we believe that both effects influence the actuator, experimental observations suggest that the stiffening impact of the electrodes is the dominating one. We base this hypothesis on the fact that similar strain-distribution profiles were observed on different devices: A high-strain region with an elongated shape (perpendicular to the feed-lines) located in the center of the actuator, and two low-strain regions located in the area where the feed-lines connect to the circular active area. This specific strain distribution seems to be induced by the electrodes geometry rather than by random variations of the membrane thickness. The membrane profile was also characterized by white light interferometry and showed thickness variations below $10 \%$. According to our simulations, such thickness variations are not enough to explain the measured strain profile.

The stiffening impact of the electrodes is usually neglected when designing and modeling DEAs, and although this is generally a valid approximation, the experimental results presented on strain distribution suggest that this design parameter cannot be neglected for thin DEAs. To support this hypothesis, we developed a simple DEA model to determine strain vs. drive voltage, taking into account the mechanical properties of the electrodes. The modeled actuator consists of an elastomer membrane under equibiaxial prestretch and sandwiched between two circular elastomer electrodes. The prestretch is modeled as a constant force acting at the periphery of the electrodes. ${ }^{22}$ The relation between the driving voltage and the actuation stretch is found by minimizing the energy function of the actuator. The electrostatic energy stored in the actuator is calculated using the energy equation of a parallel plate capacitor, and the mechanical energy stored in the membrane and the electrodes is calculated using the strain energy density function of the Gent model. ${ }^{23}$

The outcome of the model is presented in Fig. 4. The dashed lines correspond to ideal electrodes (no stiffness), and the solid lines correspond to electrodes with $2 \mu \mathrm{m}$ thickness and the same shear modulus as the membrane. Figure 4(a) presents Voltage-Strain profiles at different membrane thicknesses, and Fig. 4(b) presents the relation between the 


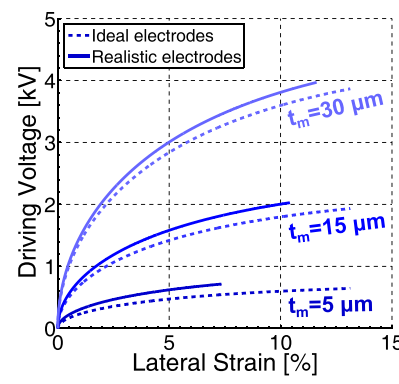

(a)

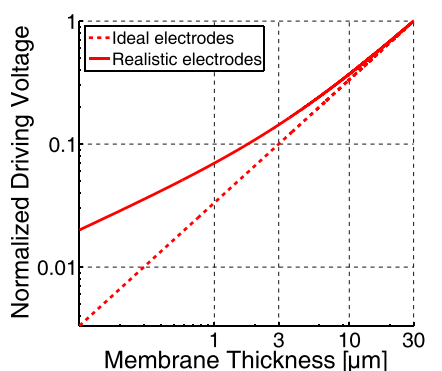

(b)
FIG. 4. Simulation results for a circular DEA under equibiaxial prestretch. (a) Voltage-Strain profiles for different membrane thicknesses $t_{m}$. The lines stop at dielectric breakdown. (b) Driving voltage (normalized to the maximum value with ideal-electrodes) as a function of the membrane thickness. The dashed lines consider ideal electrodes of zero stiffness, and the solid lines include realistic $2 \mu$ m-thick electrodes of the same shear modulus as the membrane. The solid lines show that the electrodes can cause lower actuation strains at dielectric breakdown and higher driving voltages. This is due to the relative stiffening impact of the electrodes (of constant thickness) which increases for thinner membranes. It shows that the electrodes stiffening impact is an important design parameter that should not be neglected for thin DEAs.

driving voltage (for a given strain) and the membrane thickness. For ideal electrodes, the driving voltage simply decreases linearly with the membrane thickness, and the maximum strain (limited by dielectric breakdown of the membrane) is independent of membrane thickness. In the case of realistic electrodes, the device exhibits lower maximum strain values and higher driving voltages than for ideal electrodes. This is due to the stiffening impact of the electrodes which increases the energy required to reach a given strain value. The relative stiffening impact of the electrodes increases as the membrane gets thinner. This effect is clearly visible from the maximum strain values in Fig. 4(a) and the normalized driving voltage in Fig. 4(b). Since the electrodes thickness does not scale down with the membrane thickness in our model, their relative contribution to the actuator stiffness consequently increases for thinner membranes. The electrodes stiffness is thus a critical parameter to consider when designing and optimizing thin DEAs.

In summary, we demonstrated that by reducing the thickness of the elastomer membrane to a few microns, DEAs can be operated at significantly lower voltages and still maintain good actuation performance. A lateral strain of $7.5 \%$ was reported at only $245 \mathrm{~V}$ on a $3 \mu \mathrm{m}$-thick membrane. This corresponds to an actuation of $125 \% / \mathrm{kV}^{2}$ which is the highest reported value for DEAs to date. We identified the electrode stiffness as an essential design parameter which can strongly influence actuation performance. This work presents a practical approach towards low-voltage DEAs, a critical step for the development of real world applications. In addition, the pad-printing based fabrication process presented here allows direct patterning of the silicone membrane, leading to $\mu \mathrm{m}$-thick membranes printed with any inplane geometry with a $100 \mu \mathrm{m}$ resolution. Combined with the ability to pattern stretchable electrodes using the same printing method, this enables unprecedented design flexibility for DEAs, giving a path to vertical integration in dielectric elastomer transducers.
This work was supported by the Swiss National Science Foundation Grant No. 200020_153122 and the FNS R'equip Grant No. 206021_139187.

${ }^{1}$ R. Pelrine, "High-speed electrically actuated elastomers with strain greater than 100\%," Science 287, 836-839 (2000).

${ }^{2}$ L. Maffli, S. Rosset, M. Ghilardi, F. Carpi, and H. Shea, "Ultrafast allpolymer electrically tunable silicone lenses," Adv. Funct. Mater. 25, 1656-1665 (2015).

${ }^{3}$ I. A. Anderson, T. A. Gisby, T. G. McKay, B. M. O'Brien, and E. P. Calius, "Multi-functional dielectric elastomer artificial muscles for soft and smart machines," J. Appl. Phys. 112, 041101 (2012).

${ }^{4}$ O. A. Araromi, I. Gavrilovich, J. Shintake, S. Rosset, M. Richard, V. Gass, and H. R. Shea, "Rollable multisegment dielectric elastomer minimum energy structures for a deployable microsatellite gripper," IEEE/ASME Trans. Mechatronics 20, 438-446 (2014).

${ }^{5}$ S. Shian, K. Bertoldi, and D. R. Clarke, "Dielectric elastomer based grippers for soft robotics," J. Adv. Mater. 27, 6814-6819 (2015).

${ }^{6} \mathrm{M}$. Aschwanden and A. Stemmer, "Polymeric, electrically tunable diffraction grating based on artificial muscles," Opt. Lett. 31, 2610-2612 (2006).

${ }^{7}$ S. Shian, R. M. Diebold, and D. R. Clarke, "Tunable lenses using transparent dielectric elastomer actuators," Opt. Express 21, 8669-8676 (2013).

${ }^{8}$ F. Xia, S. Tadigadapa, and Q. M. Zhang, "Electroactive polymer based microfluidic pump," Sens. Actuators, A 125, 346-352 (2006).

${ }^{9}$ P. Lotz, M. Matysek, and H. F. Schlaak, "Fabrication and application of miniaturized dielectric elastomer stack actuators," IEEE/ASME Trans. Mechatronics 16, 58-66 (2011).

${ }^{10}$ C. Murray, D. McCoul, E. Sollier, T. Ruggiero, X. Niu, Q. Pei, and D. D. Carlo, "Electro-adaptive microfluidics for active tuning of channel geometry using polymer actuators," Microfluid. Nanofluid. 14, 345-358 (2013).

${ }^{11}$ F. Carpi, D. De Rossi, R. Kornbluh, R. E. Pelrine, and P. Sommer-Larsen, Dielectric Elastomers as Electromechanical Transducers: Fundamentals, Materials, Devices, Models and Applications of an Emerging Electroactive Polymer Technology (Elsevier, 2011).

${ }^{12}$ M. V. Circu, Y. S. Ko, A. C. Gerecke, and D. M. Opris, "Soft polydimethylsiloxane thin elastomeric films by in situ polymerization to be used as dielectricum in actuators," Macromol. Mater. Eng. 299, 1126-1133 (2014).

${ }^{13}$ X. Niu, H. Stoyanov, W. Hu, R. Leo, P. Brochu, and Q. Pei, "Synthesizing a new dielectric elastomer exhibiting large actuation strain and suppressed electromechanical instability without prestretching," J. Polym. Sci., Part B: Polym. Phys. 51, 197-206 (2013).

${ }^{14}$ S. Risse, B. Kussmaul, H. Krüger, and G. Kofod, "Synergistic improvement of actuation properties with compatibilized high permittivity filler," Adv. Funct. Mater. 22, 3958-3962 (2012).

${ }^{15}$ L. J. Romasanta, P. Leret, L. Casaban, M. Hernández, M. A. de la Rubia, J. F. Fernández, J. M. Kenny, M. A. Lopez-Manchado, and R. Verdejo, "Towards materials with enhanced electro-mechanical response: $\mathrm{CaCu}_{3} \mathrm{Ti}_{4} \mathrm{O}_{12}$ polydimethylsiloxane composites," J. Mater. Chem. 22, 24705-24712 (2012).

${ }^{16}$ S. J. Dünki, Y. S. Ko, F. A. Nüesch, and D. M. Opris, "Self-repairable, high permittivity dielectric elastomers with large actuation strains at low electric fields," Adv. Funct. Mater. 25, 2467-2475 (2015).

${ }^{17}$ S. Akbari, S. Rosset, and H. R. Shea, "Improved electromechanical behavior in castable dielectric elastomer actuators," Appl. Phys. Lett. 102, 071906 (2013).

${ }^{18}$ E. Kang, J. Ryoo, G. S. Jeong, Y. Y. Choi, S. M. Jeong, J. Ju, S. Chung, S. Takayama, and S. H. Lee, "Large-scale, ultrapliable, and free-standing nanomembranes," Adv. Mater. 25, 2167-2173 (2013).

${ }^{19}$ T. Töpper, F. Weiss, B. Osmani, C. Bippes, V. Leung, and B. Müller, "Siloxane-based thin films for biomimetic low-voltage dielectric actuators," Sens. Actuators, A 233, 32-41 (2015).

${ }^{20}$ S. Rosset and H. R. Shea, "Flexible and stretchable electrodes for dielectric elastomer actuators," J. Appl. Phys. A 110, 281-307 (2013).

${ }^{21}$ S. Rosset, S. Araromi, S. Schlatter, and H. Shea, "Fabrication process of silicone-based dielectric elastomer actuators," J. Visualized Exp. (JoVE) (in press).

${ }^{22}$ J. Huang, T. Li, C. Chiang Foo, J. Zhu, D. R. Clarke, and Z. Suo, "Giant, voltage-actuated deformation of a dielectric elastomer under dead load," Appl. Phys. Lett. 100, 041911 (2012).

${ }^{23}$ A. Gent, "A new constitutive relation for rubber," Rubber Chem. Technol. 69, 59-61 (1996). 